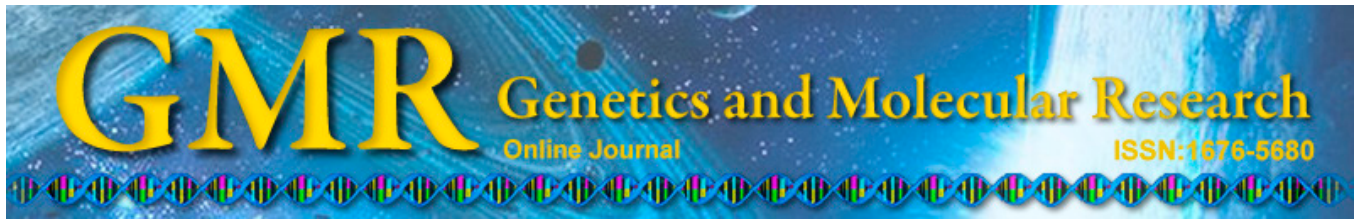

\title{
Fyn arrests swainsonine-induced apoptosis in 293T cells via Akt and its phosphorylation
}

\author{
L. An*, W.W. Li* and G.C. Cheng \\ Huaihe Hospital, Henan University, Kaifeng, Henan, China \\ *These authors contributed equally to this study. \\ Corresponding author: G.C. Cheng \\ E-mail: hndxhhyycgc@163.com
}

Genet. Mol. Res. 14 (2): 5304-5309 (2015)

Received August 28, 2014

Accepted February 5, 2015

Published May 18, 2015

DOI http://dx.doi.org/10.4238/2015.May.18.23

\begin{abstract}
Swainsonine (SW), an extract from Astragalus membranaceus, represents a new class of compounds that inhibit growth and induce apoptosis in a cancer model. In this study, we demonstrated the effect of Fyn on SW-induced apoptosis in 293T cells. Western blotting was used to measure the expression of the apoptosis-related factors caspase-3, Bcl-2, Bax, and the key factor Akt (also known as protein kinase B). Apoptosis increased dramatically after treatment with SW. Unlike the control group, after transfection with Fyn, the expression of Bcl-2, in contrast to Bax, was markedly upregulated. The results also showed that the protein expression levels of Akt and phosphorylated Akt were markedly increased. Our results establish that Fyn can arrest SW-induced apoptosis via the activity of Akt and its effective phosphorylation in 293 T cells.
\end{abstract}

Key words: Akt; Apoptosis; Caspase-3; Fyn; Swainsonine 


\section{INTRODUCTION}

It has been reported the swainsonine (SW), a natural plant alkaloid, promotes antitumor immunomodulatory activities and induces apoptosis (Oredipe et al., 2003; Li et al., 2012a). Previous studies have shown that SW enhances apoptosis in MHCC97-H cells, colorectal cancer cells, and human diffuse large B cell lymphoma (Suzuki and Abe, 2008; deFreitas-Junior et al., 2012; You et al., 2012). By upregulating Bax and downregulating Bcl-2, SW promotes apoptosis through activation of the mitochondrial pathway (Li et al., 2012b). However, the molecular mechanism of SW-induced apoptosis remains unclear, and further research into antitumor agents is required.

A member of the Src kinase family, Fyn, is expressed in a variety of tissues and is involved in apoptosis (Luciano et al., 2001; Chen et al., 2011). It has been reported that Fyn plays a critical role in cell migration, proliferation, and adhesion, and deficiency of Fyn causes apoptosis (Eguchi et al., 2012; Takadera et al., 2012). Accordingly, increasing the activity of Fyn artificially may depress the sensitivity of cells to apoptosis inducers (Chen et al., 2011), which will provide reliable information on SW-induced apoptosis for application in different fields. In addition, it has been demonstrated that the inactivation of Akt (also known as protein kinase B) may block caspase-3-mediated apoptosis (Fu et al., 2014).

In the present study, we describe the role of Fyn in apoptosis. No detailed information is available on the relationship between Fyn and SW-induced apoptosis. Therefore, we first performed an analysis of apoptosis-associated factors.

\section{MATERIAL AND METHODS}

\section{Plasmid construction}

Restriction enzymes EcoRI and SmaI were purchased from Takara (Otsu, Shiga, Japan). Plasmids encoding mouse Fyn were constructed by reverse transcription-polymerase chain reaction. The pCAG-MCS vector was used as a control. Sequences for mouse Fyn were: 5'-CGGAATTCATGGGCTGTGTGCAA-3' (forward); and 5'-ACGCGTCGACTCACAGGT TTTCACCG-3' (reverse); enzyme restriction sites are underlined.

\section{Cell culture}

The 293T cells were grown in Dulbecco's modified Eagle's medium with 1\% penicillin/streptomycin and supplemented with $10 \%$ fetal bovine serum at $37^{\circ} \mathrm{C}$ in $5 \% \mathrm{CO}_{2}$.

\section{Transfection and Western blot}

The $293 \mathrm{~T}$ cells were seeded on cell culture dishes before transfection. Using Lipofectamine 2000 reagent (Invitrogen, Life Technologies, Carlsbad, CA, USA), cells were transfected with $4 \mu \mathrm{g}$ of each plasmid according to manufacturer instructions. Assays were performed $48 \mathrm{~h}$ after transfection. Western blot analysis included lysates of $293 \mathrm{~T}$ cells. Hypotonic lysis buffer $(50 \mathrm{mM}$ Tris- $\mathrm{HCl}, 150 \mathrm{mM} \mathrm{NaCl}, 5 \mathrm{mM}$ ethylenediaminetetraacetic acid, $1 \%$ Triton X-100, $1 \%$ sodium deoxycholate, $0.1 \%$ sodium dodecyl sulfate, sodium orthovanadate, sodium fluoride, $\mathrm{pH}$ 7.4) was added to each sample. Proteins were separated by 
sodium dodecyl sulfate-polyacrylamide gel electrophoresis and transferred electrophoretically to polyvinylidene fluoride membranes. The membranes were blocked with 5\% skimmed milk in TBST (20 mM Tris- $\mathrm{HCl}, 150 \mathrm{mM} \mathrm{NaCl}$, and $0.05 \%$ Tween $20, \mathrm{pH} 8.0)$ for $1 \mathrm{~h}$. A monoclonal antibody against Bax, Bcl-2, Caspase-3, Akt, p-Akt (Millipore, Billerica, MA, USA) and Fyn (Santa Cruz Biotechnology, Santa Cruz, CA, USA) was used as primary antibody at a dilution of 1:300, followed by treatment with horseradish peroxidase-conjugated secondary antibodies and ECL Plus Western Blot detection reagents (Thermo Scientific, Waltham, MA, USA).

\section{Data analysis}

Statistical differences from three independent experiments were analyzed by the unpaired Student $t$-test using the GraphPad Prism 5 software.

\section{RESULTS}

\section{Effect of different concentrations of SW on caspase-3}

To examine the apoptosis effect, specific reductions of SW concentration in 293T cells were carried out over $24 \mathrm{~h}$. SW-induced apoptosis was mediated by activation of caspase- 3 . Western blot analysis showed that expression of caspase-3 was at its greatest at an SW concentration of $1.75 \mu \mathrm{g} / \mathrm{mL}$ (Figure 1, $\mathrm{P}<0.001$ ). Subsequent experiments were treated with 1.75 $\mu \mathrm{g} / \mathrm{mL} \mathrm{SW}$ after transfection with Fyn for $24 \mathrm{~h}$.

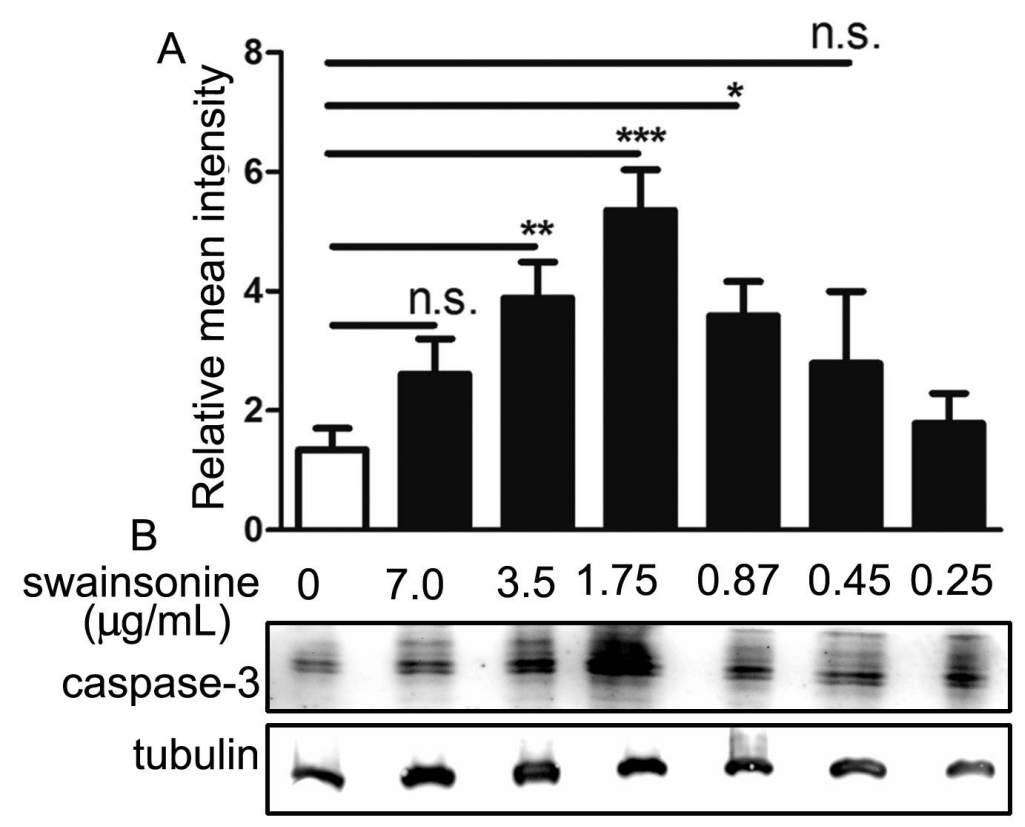

Figure 1. Effect of different doses of swainsonine on caspase-3. A. Densitometric analysis of caspase-3 in $293 \mathrm{~T}$ cells. $* \mathrm{P}<0.05,{ }^{*} \mathrm{P}<0.01,{ }^{*} * \mathrm{P}<0.001$. B. Representative Western blot of caspase- 3 from $293 \mathrm{~T}$ cells treated with swainsonine at the concentrations of $0,7.0,3.5,1.75,0.87,0.45$, and $0.25 \mu \mathrm{g} / \mathrm{mL}$. 


\section{Effect of Fyn on SW-induced expression of apoptosis-related factors}

To demonstrate the effect of Fyn on SW-induced apoptosis, we detected expression of apoptosis-related factors, i.e., Bax and Bcl-2. As shown in Figure 2, overexpression of Fyn significantly increased the expression of $\mathrm{Bcl}-2(\mathrm{P}<0.001)$ but reduced the expression of Bax $(\mathrm{P}<0.01)$ compared with the control and negative control groups. In other words, $\mathrm{SW}$-induced apoptosis was arrested by Fyn because of the expression of Bcl-2 and the inhibition of Bax. Our results show that overexpression of Fyn inhibits SW-induced apoptosis.

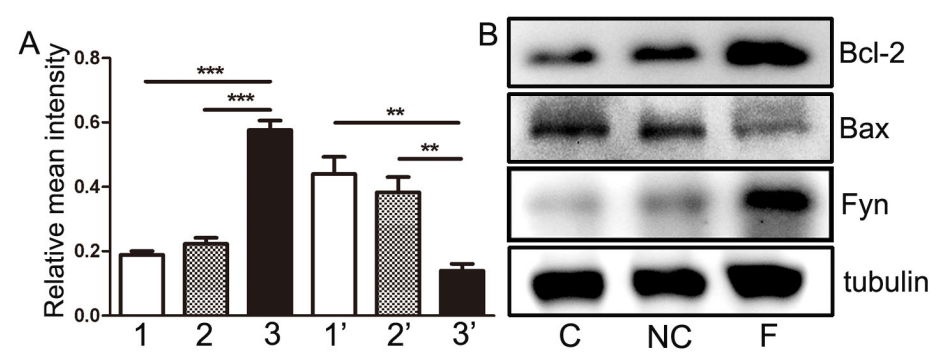

Figure 2. Effect of Fyn on the expression of Bax and Bcl-2 in 293T cells after treatment with swainsonine. Columns 1,1 ', and lane $C$ : control group; columns 2, 2', and lane $N C$ : negative control group; columns 3, 3', and lane F: Fyn group. Columns 1, 2, and 3 represent expression of Bcl-2, and 1', 2', and 3' represent expression of Bax. A. Densitometric analysis of Bcl-2 and Bax in 293T cells. P values are reported as means \pm standard error of the mean determined from three independent experiments; $* * \mathrm{P}<0.01 ; * * * \mathrm{P}<0.001$. B. Protein expression of Bcl-2 and Bax by Western blot after treatment with $1.75 \mu \mathrm{g} / \mathrm{mL}$ swainsonine for $24 \mathrm{~h}$.

\section{Overexpression of Fyn elevated phosphorylation of Akt and p-Akt to confront apoptosis}

Studies have proved that inhibition of Akt is involved in apoptosis (Zhang et al., 2013; Won et al., 2014). To identify whether Akt is involved in SW-induced apoptosis through overexpression of Fyn, we examined Akt and its phosphorylation at residue 473. The results showed that expression of Akt increased significantly after Fyn transfection $(\mathrm{P}<0.001)$. Western blot analysis also showed that the expression level of p-Akt increased significantly after Fyn transfection compared with the control group (Figure 3, P $<0.001$ ) and the negative control group (Figure 3, $\mathrm{P}<0.01$ ). The results revealed that Fyn can effectively inhibit the apoptosis effect of SW, and elevate anti-apoptosis via active Akt and its phosphorylation.

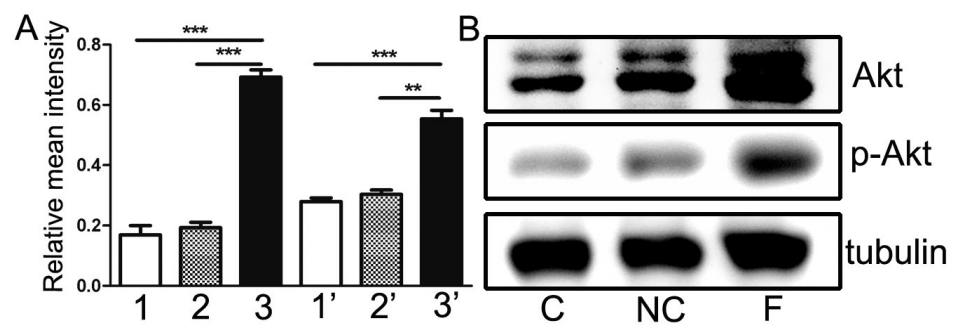

Figure 3. Effect of Fyn on the expression of Akt and its phosphorylation in 293T cells after treatment with swainsonine. Columns 1, 1', and lane $C$ : control group; columns 2, 2', and lane $N C$ : negative control group; columns 3, 3', and lane F: Fyn group. Columns 1, 2, and 3 represent expression of Akt, and 1', 2', and 3' represent expression of p-Akt. A. Densitometric analysis of Akt and p-Akt in 293T cells from three independent experiments; $* * \mathrm{P}<0.01$; $* * * \mathrm{P}<0.001$. B. Protein expression of Akt and $\mathrm{p}$-Akt by Western blot after treatment with $1.75 \mu \mathrm{g} / \mathrm{mL}$ swainsonine. 


\section{DISCUSSION}

It has been reported that SW is a useful therapy for metastasis in B-leukemic and pancreatic cancer cells (Schwarz et al., 1999; Dörrie et al., 2002), as well as Jurkat T lymphocytes (Walzel et al., 2006). It can induce apoptosis in a dose-dependent manner, but the effect of different doses of SW on the expression of caspase- 3 has been poorly investigated up to the time of the present study. Our results demonstrated that a specific dose of SW $(1.75 \mu \mathrm{g} / \mathrm{mL})$ increased the maximum expression of caspase- 3 in $293 \mathrm{~T}$ cells in a dose-response manner. The present study not only confirmed previous studies but also investigated the effect of different doses of SW on caspase-3 in 293 T cells. A previous study had demonstrated that SW induced apoptosis in a dose-dependent manner (Huang et al., 2013). In contrast, our results showed that higher/lower concentrations ( 7.0 and $0.25 \mu \mathrm{g} / \mathrm{mL}$ ) had no effect on SW-induced apoptosis, which suggests that even particularly high doses of SW may result in cell death, while a lower dose may protect against apoptosis.

$\mathrm{SW}$ is a cytokine with strong antitumor activity, and it can induce apoptosis in a variety of cells. In the normal physiological state, the expression level of Bax in cells is low, in contrast to Bcl-2, and SW decreases the expression of Bcl-2 (Sun et al., 2007). Studies have demonstrated that Fyn plays a critical role in neuronal apoptosis and is intimately correlated with apoptosis in vitro (Du et al., 2012; Eguchi et al., 2012). Here, Western blotting was used to study the expression of apoptosis-related factors, including Bax and Bcl-2, after transfection with Fyn. Our study showed that overexpression of Fyn can significantly increase the expression of the apoptosis suppressor gene Bcl-2, and downregulate expression of the apoptotic protein Bax. Based on the two diametrically opposed points above, our results strongly suggest that Fyn may be a tumor suppressor, and can inhibit SW-induced apoptosis in 293T cells.

Fyn is intimately correlated with the phosphoinositide-3-kinase/Akt signaling pathway (Lue et al., 2007; Tang et al., 2007; Holen et al., 2008) and prompts proliferation and differentiation (Kim et al., 2010). Akt plays an important role in cell survival and the development of cancer (Brauer and Tyner, 2009). Therefore, Akt is regarded as an apoptosis suppressor factor and was used in our study. As in a previous study (Yu et al., 2013), our study showed that overexpression of Fyn can significantly increase Akt and p-Akt treated with SW. The results indicate that Fyn can promote the activation of Akt and then protect against apoptosis by regulating the expression of Akt phosphorylation.

In summary, we have shown that Fyn expression elevates anti-apoptosis by inhibiting the expression of Bax and regulating the expression of Bcl-2. Fyn effectively inhibited SWinduced apoptosis in 293T cells via Akt and its phosphorylation. However, the question of whether Fyn participates in SW-induced apoptosis in other ways requires further investigation.

\section{REFERENCES}

Brauer PM and Tyner AL (2009). RAKing in AKT: a tumor suppressor function for the intracellular tyrosine kinase FRK. Cell Cycle 8: 2728-2732.

Chen ZY, Cai L, Zhu J, Chen M, et al. (2011). Fyn requires HnRNPA2B1 and Sam68 to synergistically regulate apoptosis in pancreatic cancer. Carcinogenesis 32: 1419-1426.

de-Freitas-Junior JC, Bastos LG, Freire-Neto CA, Rocher BD, et al. (2012). N-glycan biosynthesis inhibitors induce in vitro anticancer activity in colorectal cancer cells. J. Cell Biochem. 113: 2957-2966.

Dörrie J, Sapala K and Zunino SJ (2002). Interferon-gamma increases the expression of glycosylated CD95 in B-leukemic cells: an inducible model to study the role of glycosylation in CD95-signalling and trafficking. Cytokine 18: 98-107. 
Du CP, Tan R and Hou XY (2012). Fyn kinases play a critical role in neuronal apoptosis induced by oxygen and glucose deprivation or amyloid-beta peptide treatment. CNS Neurosci. Ther. 18: 754-761.

Eguchi R, Kubo S, Takeda H, Ohta T, et al. (2012). Deficiency of Fyn protein is prerequisite for apoptosis induced by Src family kinase inhibitors in human mesothelioma cells. Carcinogenesis 33: 969-975.

Fu Z, Zou F, Deng H, Zhou H, et al. (2014). Estrogen protects SGC7901 cells from endoplasmic reticulum stress-induced apoptosis by the Akt pathway. Oncol. Lett. 7: 560-564.

Holen HL, Shadidi M, Narvhus K, Kjøsnes O, et al. (2008). Signaling through ephrin-A ligand leads to activation of Src-family kinases, Akt phosphorylation, and inhibition of antigen receptor-induced apoptosis. J. Leukoc. Biol. 84: $1183-1191$.

Huang Y, Li W, Zhao X, Ding L, et al. (2013). Swainsonine differentially affects steroidogenesis and viability in caprine luteal cells in vitro. Theriogenology 80: 41-49.

Kim HJ, Warren JT, Kim SY, Chappel JC, et al. (2010). Fyn promotes proliferation, differentiation, survival and function of osteoclast lineage cells. J. Cell Biochem. 111: 1107-1113.

Li Z, Huang Y, Dong F, Li W, et al. (2012a). Swainsonine promotes apoptosis in human oesophageal squamous cell carcinoma cells in vitro and in vivo through activation of mitochondrial pathway. J. Biosci. 37: 1005-1016.

Li Z, Xu X, Huang Y, Ding L, et al. (2012b). Swainsonine activates mitochondria-mediated apoptotic pathway in human lung cancer A549 cells and retards the growth of lung cancer xenografts. Int. J. Biol. Sci. 8: 394-405.

Luciano F, Ricci JE and Auberger P (2001). Cleavage of Fyn and Lyn in their N-terminal unique regions during induction of apoptosis: a new mechanism for Src kinase regulation. Oncogene 20: 4935-4941.

Lue H, Thiele M, Franz J, Dahl E, et al. (2007). Macrophage migration inhibitory factor (MIF) promotes cell survival by activation of the Akt pathway and role for CSN5/JAB1 in the control of autocrine MIF activity. Oncogene 26: 5046-5059.

Oredipe OA, Furbert-Harris PM, Laniyan I, Green WR, et al. (2003). Enhanced proliferation of functionally competent bone marrow cells in different strains of mice treated with swainsonine. Int. Immunopharmacol. 3: 445-455.

Schwarz RE, Wojciechowicz DC, Picon AI, Schwarz MA, et al. (1999). Wheatgerm agglutinin-mediated toxicity in pancreatic cancer cells. Br. J. Cancer 80: 1754-1762.

Sun JY, Zhu MZ, Wang SW, Miao S, et al. (2007). Inhibition of the growth of human gastric carcinoma in vivo and in vitro by swainsonine. Phytomedicine 14: 353-359.

Suzuki $\mathrm{O}$ and Abe M (2008). Cell surface N-glycosylation and sialylation regulate galectin-3-induced apoptosis in human diffuse large B cell lymphoma. Oncol. Rep. 19: 743-748.

Takadera T, Fujibayashi M, Koriyama Y and Kato S (2012). Apoptosis induced by SRC-family tyrosine kinase inhibitors in cultured rat cortical cells. Neurotox. Res. 21: 309-316.

Tang X, Feng Y and Ye K (2007). Src-family tyrosine kinase fyn phosphorylates phosphatidylinositol 3-kinase enhanceractivating Akt, preventing its apoptotic cleavage and promoting cell survival. Cell Death Differ. 14: 368-377.

Walzel H, Fahmi AA, Eldesouky MA, Abou-Eladab EF, et al. (2006). Effects of N-glycan processing inhibitors on signaling events and induction of apoptosis in galectin-1-stimulated Jurkat T lymphocytes. Glycobiology 16: 12621271.

Won KJ, Kim BK, Han G, Lee K, et al. (2014). NSC126188 induces apoptosis of prostate cancer PC-3 cells through inhibition of Akt membrane translocation, FoxO3a activation, and RhoB transcription. Apoptosis 19: 179-190.

You N, Liu W, Wang T, Ji R, et al. (2012). Swainsonine inhibits growth and potentiates the cytotoxic effect of paclitaxel in hepatocellular carcinoma in vitro and in vivo. Oncol. Rep. 28: 2091-2100.

Yu L, Lin Q, Feng J, Dong X, et al. (2013). Inhibition of nephrin activation by c-mip through Csk-Cbp-Fyn axis plays a critical role in Angiotensin II-induced podocyte damage. Cell Signal. 25: 581-588.

Zhang Y, Sun S, Chen J, Ren P, et al. (2013). Oxymatrine induces mitochondria dependent apoptosis in human osteosarcoma MNNG/HOS cells through inhibition of PI3K/Akt pathway. Tumour Biol. 35: 1619-1625. 